\title{
Design and implementation of laser based intelligent embedded dual modes cruise control system using FPGA
}

\author{
Ow Hui Nee, Muataz H. Salih, Chin Beng Lim \\ School of Computer and Communication Engineering, Universiti Malaysia Perlis (UniMAP), Malaysia
}

\begin{tabular}{l} 
Article Info \\
\hline Article history: \\
Received Sep 21, 2018 \\
Revised Dec 1, 2018 \\
Accepted Dec 25, 2018 \\
\hline
\end{tabular}

\section{Keywords:}

Cruise control

Dual modes

Fields Programmble Gate Array (FPGA)

\begin{abstract}
Cruise control system is also known as speed control which use to take over the car from the driver and stabilize the car with the speed had been set. It is one of the important elements in the intelligent vehicles. Currently, driver has to accelerate the car until certain speed in order to activate cruise control system. After it had been activated, driver still needs to put on attention to decelerate the car when there is another one in front and possibility to get accident is high. To overcome this problem and enhance current cruise system, this project has two laser-based working modes. First one is normal mode that following the same current cruise system procedure. Second is pre-set mode that allow driver to enter required speed without necessity to use petrol pedal. Both modes will use laser transceivers to add on perception feature to cruise control system. The system is designed and implemented using FPGA as main processing technology. Also, Quartus II 13.0 SP is used and DE0-Nano board is utilized as project platform. The input management is implemented to handle all sensors data and feed main processing unit with proper data stream. The result is efficient in term of system response, accurate cruise control and trustable design as well. The implement system is achieved up to $1.3 \mathrm{GHz}$ and 1,337 logic elements are consumed.
\end{abstract}

Copyright () 2019 Institute of Advanced Engineering and Science. All rights reserved.

\section{Corresponding Author:}

Ow Hui Nee,

School of Computer and Communication Engineering,

Universiti Malaysia Perlis(UniMAP),

First Floor, Pauh Putra Campus, 02600 Arau, Perlis, Malaysia.

Email: hannahow984@gmail.com

\section{INTRODUCTION}

Cruise control, also known as speed control system which will automatically control the speed of the vehicle and take over the throttle of the vehicle and maintain the desired speed set by the driver [1]. It is widely use in the Advance Driving Assistance System (ADAS) [2]. This cruise control system has many advantages, such as improving traffic flow, providing safety to the driver and passenger, it also increases fuel efficiency and at the same time giving the user's feet on a rest in a long journey road [3-8].

Traditional cruise control keep a constant speed set by the driver and the cruise will be stopped when the brake pedal is press by the driver, change gear, or the cruise control had been turn OFF [9]. The cruise control usually will only activate when the minimum speed limit is reached. According to N. Vedam [10], a cruise control can only be activated when a car exceeds approximate $25 \mathrm{mph}(40 \mathrm{~km} / \mathrm{h})$. However, different type of the car produce by the industries may have different minimum speed for cruise control system activation. Beside traditional cruise control, Adaptive Cruise Control (ACC) and Cooperative Adaptive Cruise Control (CACC) also part of the development from the traditional cruise control to advance the ADAS technology [11]. The automatic driving takes over by the cruise control system become part of importance for future generation of cars [12].

In this paper, the laser based intelligent embedded dual mode cruise control system is designed and implemented. Beside the normal mode of the cruise control, the pre-set mode is added in the cruise control 
system so user can use both modes when driving [13]. Pre-set mode mean the driver can pre-set the desired speed wish to maintain so the cruise system will automatically accelerate, decelerate or maintain the speed based on the data receive. This project is designed using the Field Programmable Gate Array (FPGA). The DE0-Nano FPGA board is use as the project platform. DE0-Nano is re-configurable, high performance and low power consumption. FPGA is used in this project due to it is low cost, flexible, low power consumption and scalable $[13,14]$.

\section{RESEARCH METHOD}

Cruise control is one of the subsystems in the Advance Driving Assistance System (ADAS) and it is widely use in present automotive vehicle technologies. Cruise control is very important in the automotive vehicle to avoid accident happen, reduce fuel consumption and increase comfortable of the driver and passengers. However, traditional cruise control only can be active when the speed of the vehicle reaches certain limit of the speed. From analysis of the literature review there is various approach of the cruise control design. By this duals modes cruise control system, user will be able to select either traditional mode or pre-set mode to activate the cruise control system. By using pre-set mode, driver could set the desired speed, and the system will then automatically accelerate or decelerate the car to this speed. In addition to this, this system will also automatically decelerate if there is any obstacle in front, or send a warning signal to the driver if there is a car sticking behind. A laser-based intelligent embedded dual modes cruise control system using FPGA is designed and implemented. Furthermore, laser-based intelligent embedded dual modes cruise control design using the DE0-Nano FPGA provides low cost, low power consumption, flexible and scalable. Besides that, FPGA also commonly use in education, research study as well as in industries fields.

DE0-Nano FPGA with Altera Cyclone IV which contain 22320 logic elements. It consists 32MB SDRAM, 2kb I2C EEPROM and 64Mb serial configuration memory devices. There are two 40 pins GPIO which consists of two $5 \mathrm{~V}$ power pin, two $3.3 \mathrm{~V}$ power pin and four ground pins. The National Semiconductor 8 channel 12bits A/D Converter allows to connect with various sensors as shown in Figure 1.

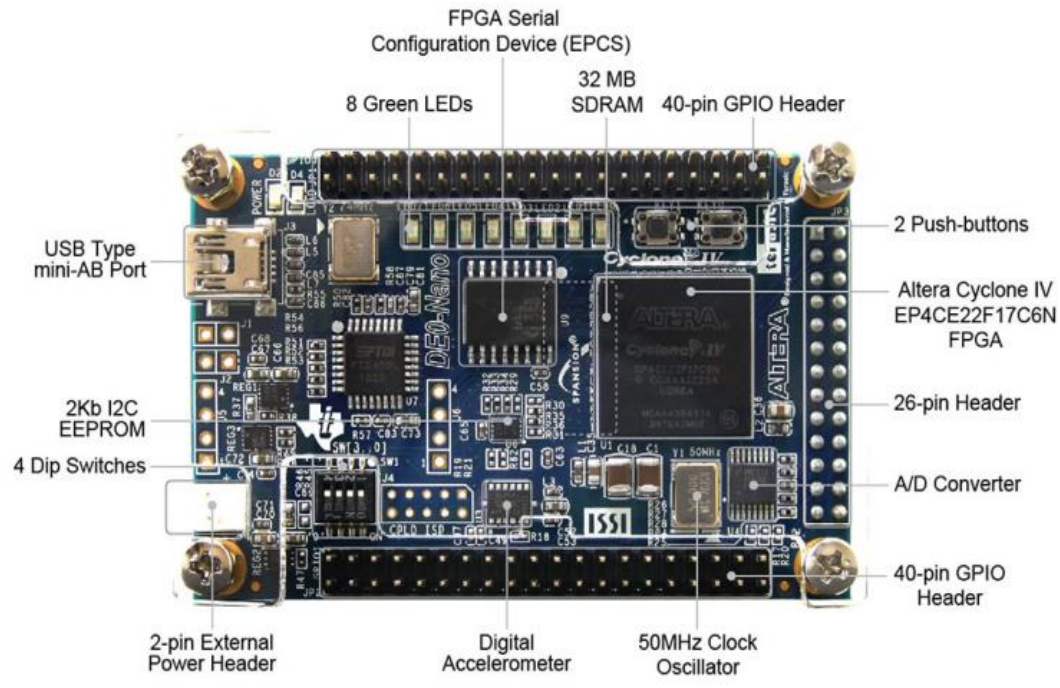

Figure 1. Top view of the DE0-Nano Board

\subsection{System flow}

The overall system flow starts with the activation of the cruise control system. Once the cruise control system is ON it will act as interrupt mode which take over the throttle for a period until it been turn OFF. When the cruise control system is turn ON, there will be two modes allow the user to choose which mode they want to activate.

The two modes of the cruise control system are the normal mode or the traditional mode and the preset mode. Traditional mode is the cruise system need to use pedal to increase speed until the desired speed user wish to set. The acceleration and deceleration of the speed will be controlled by cruise based on the data of laser transceiver and the speed of vehicle. 
Pre-set mode cruise control allow user to pre-set the desire speed once the cruise control is turn ON. Based on the desired speed the cruise system will accelerate and decelerate the speed when necessary. The data from laser transceiver sensor will received to generate the accurate action without any delay of the information. The cruise system will be deactivated when user turn OFF the cruise control system or the brake pedal is pressed.

\subsection{Top level design}

Top level design is an overview of the system which described the function of each element block contain in the system. The specific operation of the block and the flow of the system is identified. Top level design is the high-level architecture of the system which is the important part of a project development and determines the relation between each modules and function in the system. The data flow, data structure also includes in the architecture

From Figure 2, the top-level design of the system is mainly build up by the sensors and keypad block as input and LCD as output block. Inside the FPGA chip there are 4 main blocks which need to execute from input to processing unit then to output. All the input and output is connected using GPIO pins in the DE0-Nano FPGA board. The input data from sensor is process by A/D Converter chip in the FPGA board and data is send to the sensor and input reader process unit trigger by multiple clock generator. The data process by the ADC is the key point for the processing unit which consists of control sub unit and cruise controller unit. Control sub unit is using to control the cruise controller for make the accurate decision such as accelerate or decelerate the speed. Speed generator is to generate the necessary speed based on the data from sensor and cruise controller decision.

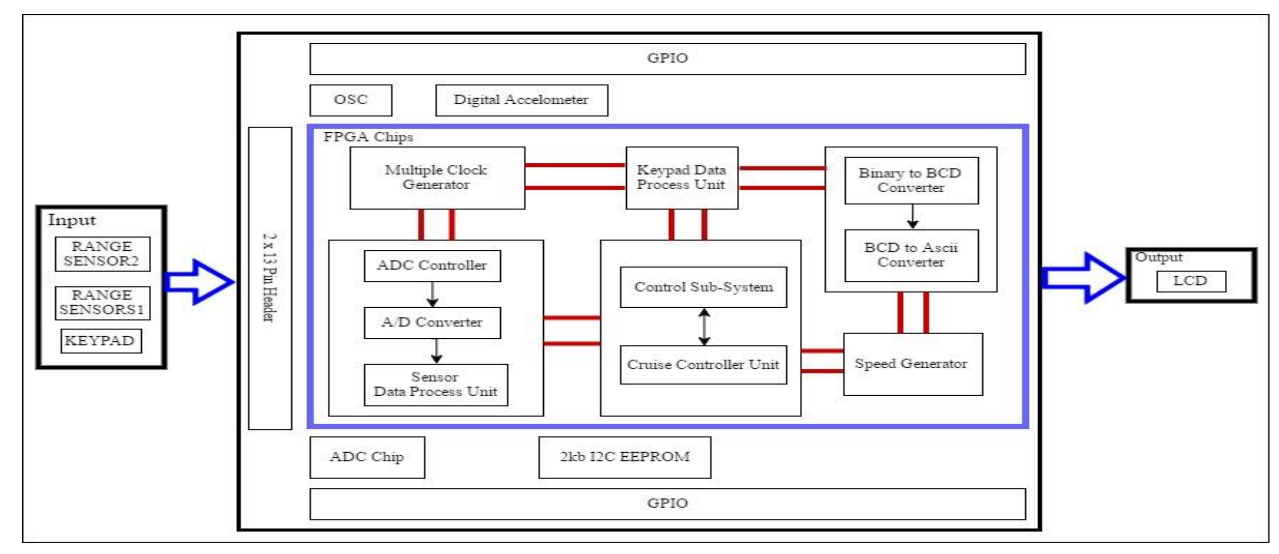

Figure 2. Top level design of the system

\subsection{Sensor Testing}

Sensor used in this project is range sensor. Before it is used, it need to be tested in term of its sensitivity and functionality based on the data sheet of the sensor. Signal conditional circuit is built for the sensor to ensure it function well and provide accurate and precise value compared to the theoretical value than had been calculated. The possible result and various condition of sensor are tested and prove by oscilloscope.

\subsection{Main Processing Unit}

The main processing unit is designed using Quartus II 13.0 Software and VHSIC Hardware Description Language (VHDL) coding. Quartus 13.0 allow to design the mega cores by using the CAD Tool.

In this project, the objective of the main processing unit is based on the input data receive then process and generate the correct decision and deliver to the output module for display. Each processing unit have different function. ADC Controller unit is to process the analogue data provide by the range sensor and the data is undergoing the calculation process to generate accurate data.in the sensor and input processing unit. At the last stage, all the result is displayed in the display module.

\subsection{Interface with DE0-Nano FPGA board}

The result of the project is shown on $2 \times 16$ LCD which is attached to the DE0-Nano Board. The speed and the cruise mode are displayed in the LCD. 


\section{RESULTS AND DISCUSSION}

\subsection{Range Sensor Testing Result}

Range sensor by model GP2D120XJ00F shown in Figure 3 is suitable for the distance measurement and has better performance than IP alternatives. The single analog output cable is connected to the analog-todigital (ADC) convertor for reading distance measurementthis.

The sensor is set up as shown in Figure 4 and tested using oscilloscope and the result is shown in Table 1. Sensitivity of different reflection object was different. Environment factor such as light condition for surrounding, reflective rate, surrounding temperature, object surface characteristics and voltage supply to the sensor may affect the sensing range of the range sensor.

As shown in Figure 5, white object has higher sensitivity than other colors object. The offset voltage of the semitransparent object less than other three colors object which is around $2.5 \mathrm{~V}$. The output voltage of the white object change over distance is from $2.875 \mathrm{~V}$ to $0.25 \mathrm{~V}$ while the semitransparent object output voltage changes from $2.5 \mathrm{~V}$ to $0.1875 \mathrm{~V}$.

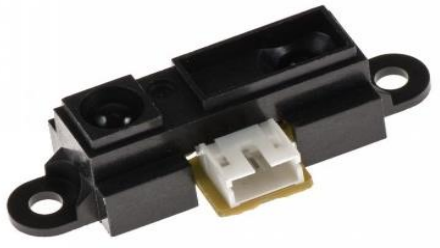

Figure 3. Range sensor

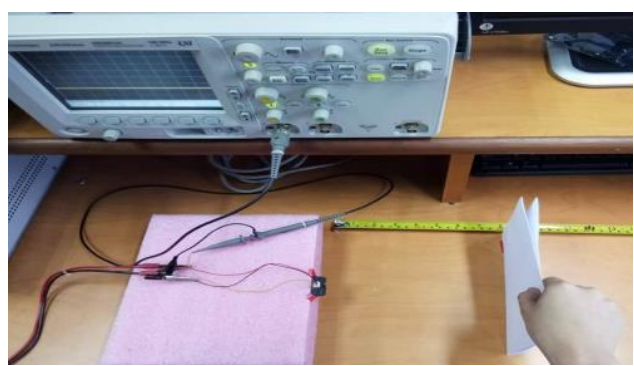

Figure 4. Range sensor set up for testing

Table 1. Output redult testes with different distance with reflective object

\begin{tabular}{|c|c|c|c|c|}
\hline \multirow{2}{*}{$\begin{array}{l}\text { Distance } \\
(\mathrm{cm})\end{array}$} & \multicolumn{4}{|c|}{ Oscilloscope Value (Voltage, V) } \\
\hline & White & Red & Black & Semi Transparent \\
\hline 0 & 0 & 0 & 0 & 0 \\
\hline 1 & 1.25 & 1.125 & 2.125 & 2.5 \\
\hline 3 & 2.875 & 2.875 & 2.8125 & 2.5 \\
\hline 5 & 2.25 & 2.125 & 2.25 & 1.6875 \\
\hline 7 & 1.6875 & 1.6625 & 1.625 & 1.3125 \\
\hline 9 & 1.3125 & 1.3125 & 1.25 & 1 \\
\hline 10 & 1.1875 & 1.125 & 1.1875 & 0.9375 \\
\hline 12 & 1 & 1 & 0.9375 & 0.75 \\
\hline 15 & 0.8125 & 0.75 & 0.6875 & 0.625 \\
\hline 20 & 0.625 & 0.625 & 0.5 & 0.375 \\
\hline 25 & 0.5 & 0.5 & 0.375 & 0.3125 \\
\hline 30 & 0.375 & 0.375 & 0.3125 & 0.25 \\
\hline 35 & 0.3125 & 0.3125 & 0.25 & 0.1875 \\
\hline 40 & 0.25 & 0.25 & 0.25 & 0.1875 \\
\hline
\end{tabular}

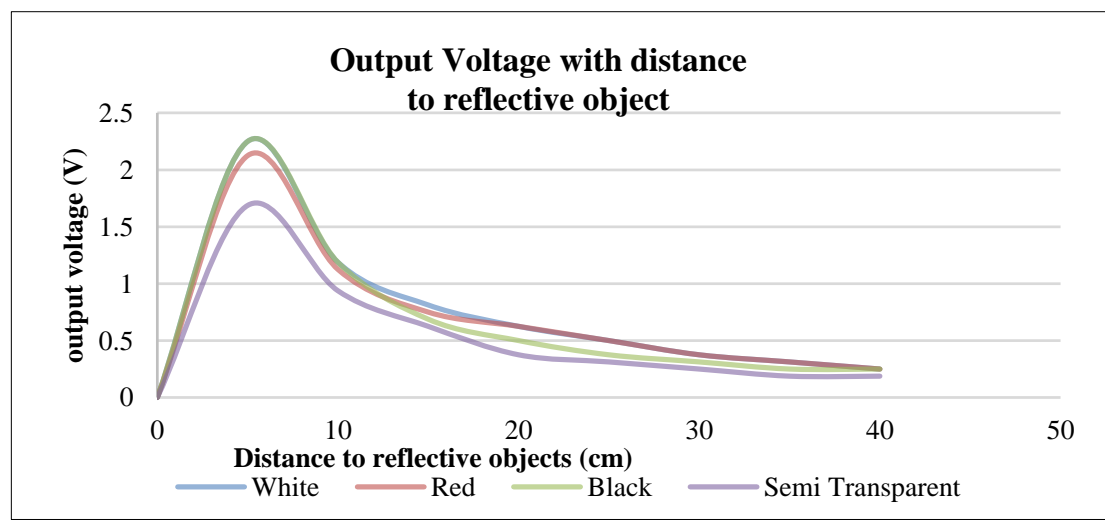

Figure 5. Graph of the output voltage with distance to reflective object 
The result of the range sensor use to control the acceleration and deceleration of the car was shown in Figure 6. by using In System Memory CAD Tools to make sure the range sensor able to provide accurate sensing result.

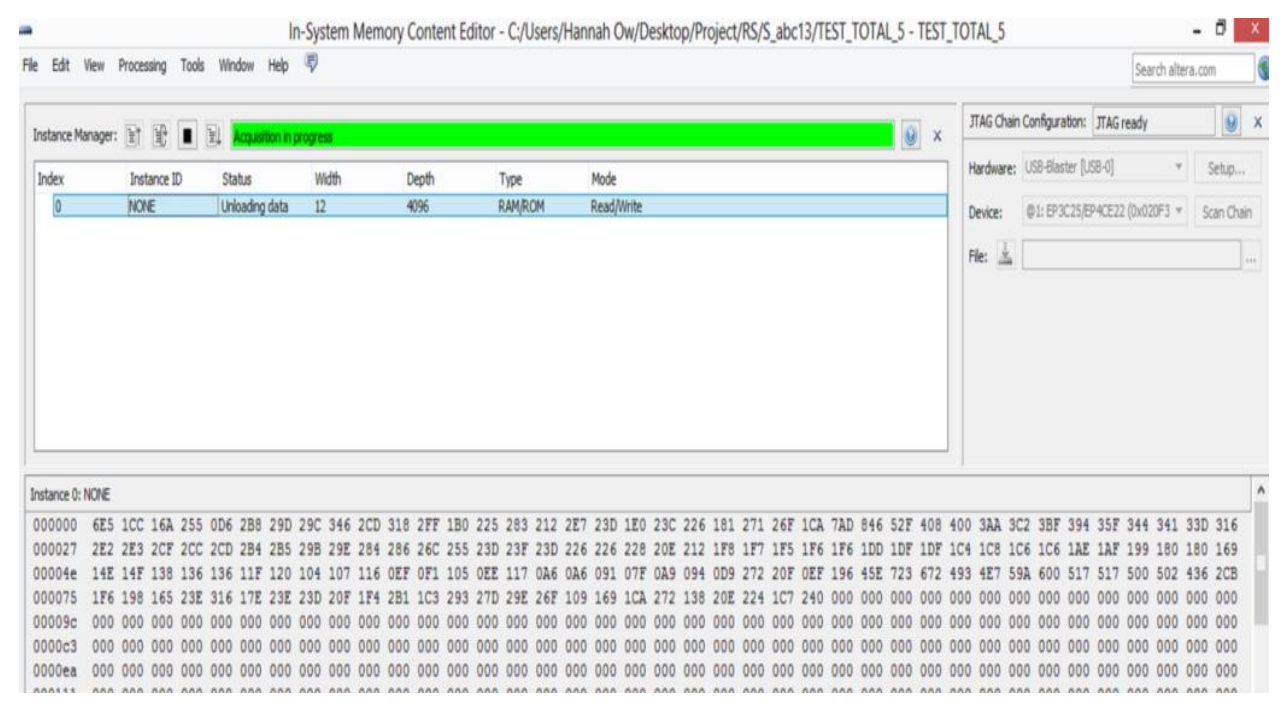

Figure 6. Result of range sensor tested by in system memory

\subsection{Speed Generator}

By using pulse width modulation (PWM) method, the speed is scaling according the required speed, as shown in Table 2. Five speed are generated for according to the capabilities of the motor of the car.

Table 2. Modulation of the speed

\begin{tabular}{ccc}
\hline Speed & Timing (second) & \\
& ON & OFF \\
\hline Low & 0.05 & 0.01 \\
Low Medium & 1 & 0.05 \\
Medium & 1.5 & 0.05 \\
High Medium & 2 & 0.05 \\
High & 3 & 0.1 \\
\hline
\end{tabular}

\subsection{Keypad}

Keypad module is designed and used to enter the desired speed as shown in Table 3. Based on the speed generated, only five inputs are designed with every 20 per scale.

Table 3. Modulation of the speed

\begin{tabular}{cc}
\hline Key & Output $(\mathrm{km} / \mathrm{h})$ \\
\hline 1 & 40 \\
2 & 60 \\
3 & 80 \\
4 & 100 \\
5 & 120 \\
\hline
\end{tabular}

\subsection{LCD Display}

External LCD is attached and interfaces with the DE0-Nano FPGA board to show the current speed and cruise set speed of the car. The input speed from the keypad also will show in the LCD before it set as the cruise speed. The cruise set speed will only show when the cruise is turn ON as shown in Figure 7 , Figure 8 and Figure 9. 


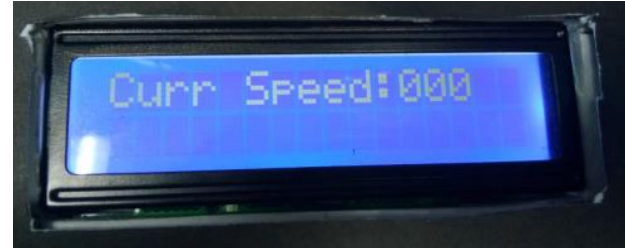

Figure 7. Current speed shown on the LCD.

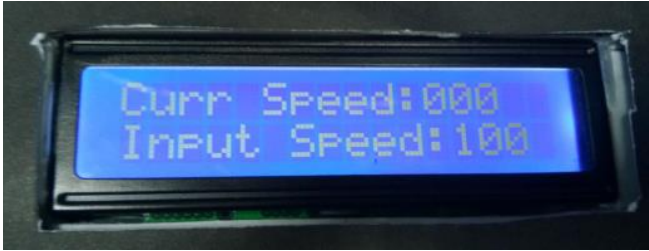

Figure 8. The keypad input speed shown at the second line of the LCD

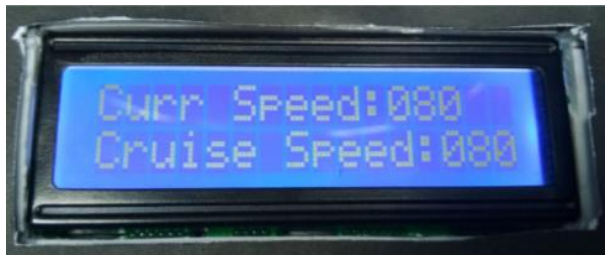

Figure 9. Current speed and cruise speed shown when cruise is turn ON.

\subsection{Prototype}

The range sensor is fixed in the front and back of the prototype as shown in the Figure 10 and Figure 11 was attached with range sensors, one in front of the car and one at the back of the car. The DE0Nano board was the main platform to control the car. Keypad attached on the dashboard was used to enter the desired speed in addition to LED indicator to alert for approaching cars from behind as shown in Figure 12 and Figure 13.

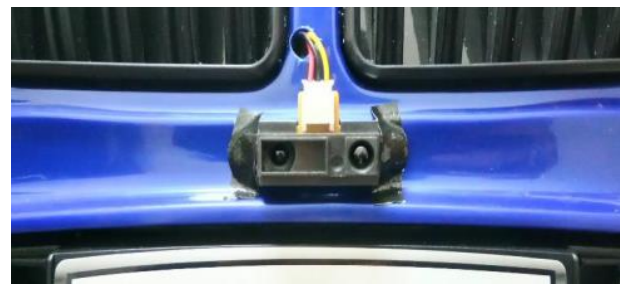

Figure 10. Range sensor in front of the car

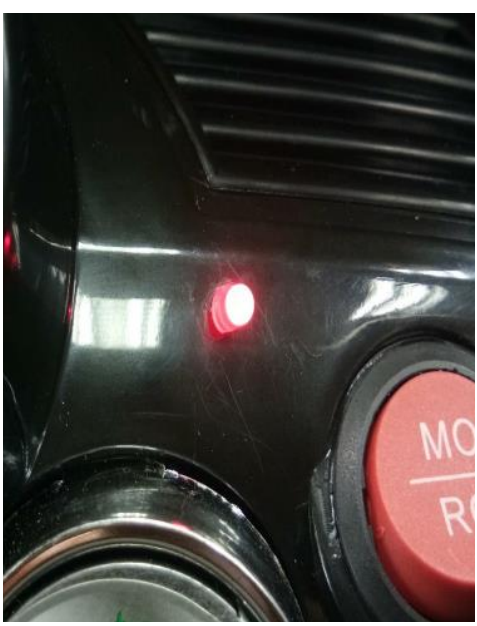

Figure 12. LED for aleart when there is car approaching at behind

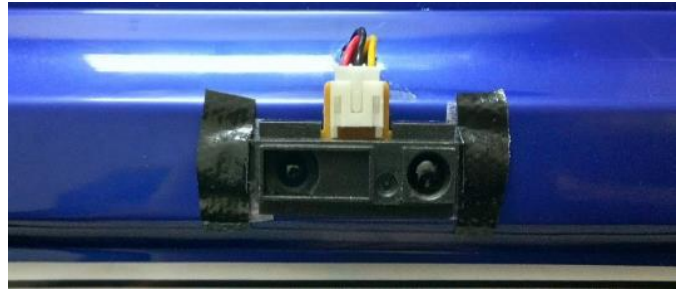

Figure 10. Range sensor at the back of the car

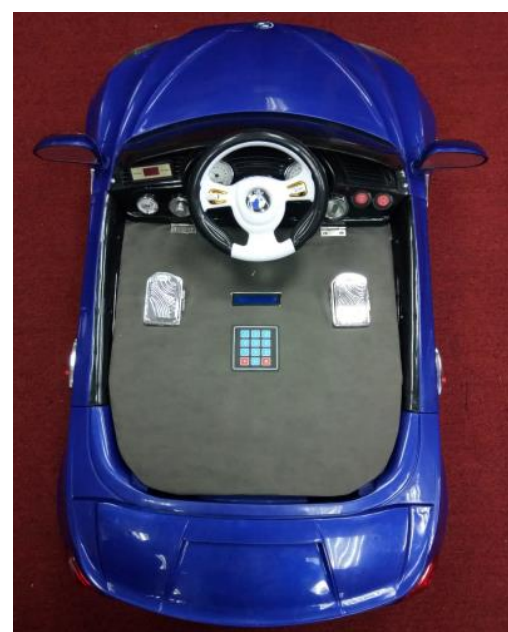

Figure 13. Complete prototype 


\subsection{Overall System Result}

All the sensors and components are successfully interfaced with the DE0-Nano FPGA. Furthermore, the architecture described are successfully implemented and shown the system able to performance as it been designed. The project is developed by using the software development tool, Quartus II 13.0 SP and the full compilation of the project is shown without error as shown in the Figure 14. This project has been used 1,337 logic elements and is the $6 \%$ for the completed project.

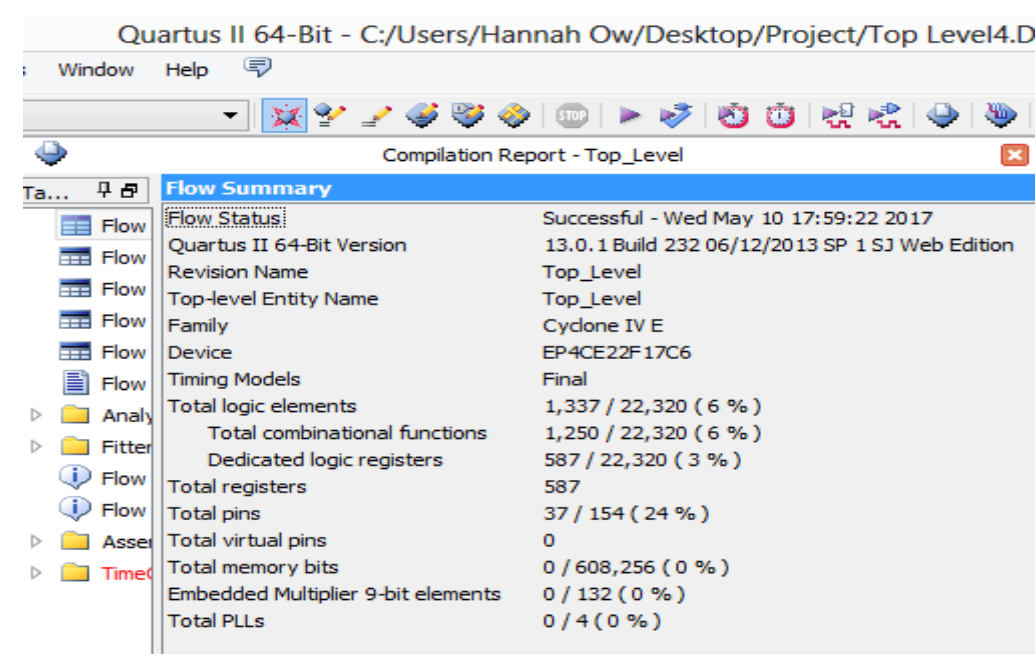

Figure 14. Full compilation report without error

\subsection{Discussion}

Speed use in this project was not the actual speed as the real vehicle performs. The speed was generated by using the speed generator with the concept of scaling using pulse width modulation to generate different speed based on the motor of the platform.

Due to the limitation of platform, only five different speeds were generated. By using keypad, user could set the speed from $40 \mathrm{~km} / \mathrm{h}$ to $120 \mathrm{~km} / \mathrm{h}$ for the speed with each scale of 20 . For this project only five input speed were perform based on the speed of the speed generator provided.

\section{CONCLUSION}

In conclusion, the dual modes cruise control system was designed and implemented to fulfill the input speed as the user need which using both keypad and pedal. The car speed able to accelerate and decelerate based on the data received from the range sensor and the cruise control speed. The alert to driver when behind vehicle is approaching also included in the project.

\section{ACKNOWLEDGEMENTS}

The authors would like to thank the Ministry of Education Malaysia (MOE) for providing the FRGS research grant (Grant no. 9003-00474) and School of Computer and Communication Engineering, University Malaysia Perlis (UniMAP) for the support.

\section{REFERENCES}

[1] Green Ron, "Five Challenges to FPGA-Based Prototyping | EE Times," S2C Inc, 2014. [Online]. Available: http://www.eetimes.com/author.asp?section_id=36\&doc_id=1324000.

[2] F. Vahid and T. Givargis, "Embedded System Design: A Unified Hardware/Software Approach,” p. 103, 1999.

[3] İ. Kılıç, A. Yazıcı, Ö. Yıldız, M. Özçelikors, and A. Ondoğan, "Intelligent Adaptive Cruise Control System Design and Implementation," Syst. Syst. Eng. Conf. (SoSE), 2015 10th, pp. 232-237, 2015.

[4] L. Luo, J. Chen, and F. Zhang, "Integrated Adaptive Cruise Control Design Considering the Optimization of Switching Between Throttle and Brake," no. Iv, pp. 1162-1167, 2016.

[5] "Types of sensors for target detection and tracking Into Robotics," 2013. [Online]. Available: https://www.intorobotics.com/types-sensors-target-detection-tracking/. 
[6] K. Konolige, J. Augenbraun, N. Donaldson, C. Fiebig, and P. Shah, “A Low-Cost Laser Distance Sensor ,” pp. 3002-3008, 2008.

[7] M. Schuster and J. Reuter, "Target Tracking in Marine Environment using Automotive Radar and Laser Range Sensor," pp. 965-970, 2015.

[8] C. Qiu, "A Design of Automobile Cruise Control System Based on Fuzzy PID," Inf. Sci. Electron. Electr. Eng. (ISEEE), 2014 Int. Conf., vol. 1, no. M, pp. 450-452, 2014.

[9] P. Maji, S. K. Patra, and K. Mahapatra, "Implementation of FPGA based fuzzy PI approximate control for automatic cruise control system," Proc. Int. Conf. Circuits, Commun. Control Comput. I4C 2014, no. November, pp. 203-206, 2014.

[10] N. Vedam, I. Diaz-Rodriguez, and S. P. Bhattacharyya, "A novel approach to the design of controllers in an automotive cruise-control system," IECON Proc. (Industrial Electron. Conf., vol. 40th Annua, pp. 2927-2932, 2014.

[11] B. Wang, D. Zhao, C. Li, and Y. Dai, "Design and implementation of an adaptive cruise control system based on supervised actor-critic learning," 2015 5th Int. Conf. Inf. Sci. Technol., pp. 243-248, 2015.

[12] N. Bekiaris-liberis, C. Roncoli, and M. Papageorgiou, "Predictor-Based Adaptive Cruise Control Design," in 2016 IEEE Conference on Control Applications (CCA), 2016, pp. 97-102.

[13] "Phase-Locked Loop Basics, PLL." [Online]. Available: https://www.altera.com/support/supportresources/operation-and-testing/pll-and-clock-management/pll-basics.html.

[14] "Sharp GP2Y0A41SK0F Analog Distance Sensor 4-30cm Jaycon Systems LLC." [Online]. Available: http://www.jayconsystems.com/sharp-gp2y0a41sk0f-analog-distance-sensor-4-30cm.html. [Accessed: 03-Apr2017].

\section{BIOGRAPHIES OF AUTHORS}

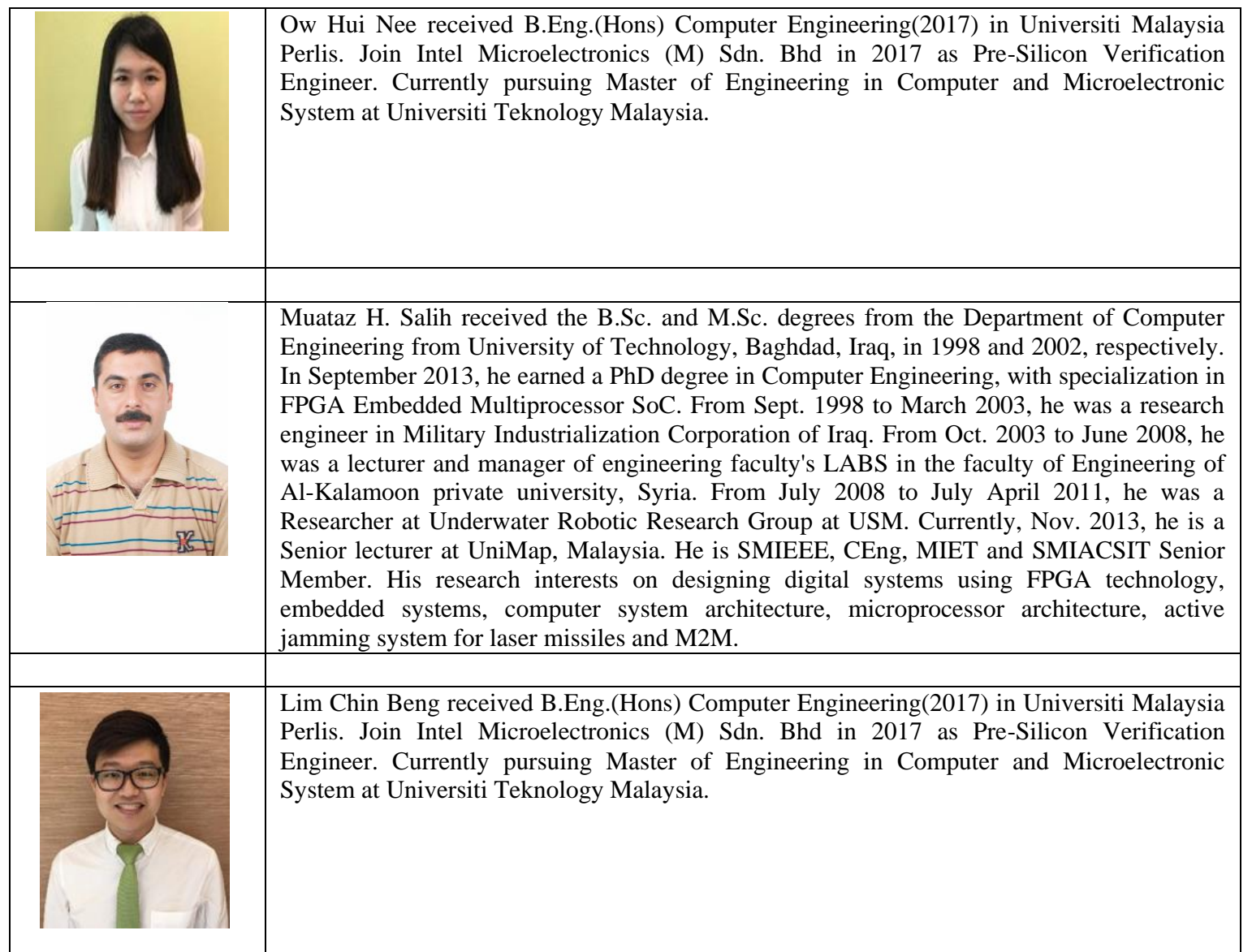

